\title{
Spectroscopie optique dans l'ultraviolet de cristaux dopés par l'ion $\mathrm{Nd}^{3+}$.
}

\author{
A. Collombet ${ }^{1}$, Y. Guyot ${ }^{1}$, E. Descroix ${ }^{2}$ et M.-F. Joubert ${ }^{1}$ \\ ${ }^{1}$ Laboratoire de Physico-Chimie des Matériaux Luminescents, \\ Université Claude Bernard-Lyon 1, UMR 5620 CNRS, \\ F-69622 VILLEURBANNE CEDEX, FRANCE \\ ${ }^{2}$ Laboratoire de Traitement du Signal et Instrumentation, \\ Université Jean Monnet, UMR 5516 CNRS, \\ F-42023 SAINT-ÉTIENNE, FRANCE \\ collombet@pcml.univ-lyon1.fr
}

Résumé. Ce travail présente les spectres d'émission polarisés dans l'ultraviolet (UV), entre 190 et $300 \mathrm{~nm}$, de cristaux dopés par l'ion $\mathrm{Nd}^{3+}$. Nous discutons des possibilités d'excitation en deux étapes des états $4 f^{2} 5 d$ de l'ion $\mathrm{Nd}^{3+}$ dans ces matrices.

Mots-clés. Spectroscopie, cristaux laser, $\mathrm{Nd}^{3+}$, transitions $4 f-5 d$, ultraviolet.

\section{INTRODUCTION}

Le développement de lasers UV, performants et compacts, pourrait profiter à de nombreux domaines tels que la biochimie, la microélectronique, l'imagerie, l'identification de composés bactériologiques, etc... Pour un champ d'applications aussi vaste et varié, il est indispensable de disposer de sources laser simples d'utilisation et permettant de couvrir une large plage spectrale dans l'ultraviolet. Les sources laser UV disponibles actuellement, telles que les lasers excimères, les lasers à solide du type YAG:Nd triplé, quadruplé ou même quintuplé en fréquence et les oscillateurs paramétriques optiques $(\mathrm{OPO})$, permettent d'accéder à un domaine spectral important mais constituent des systèmes encore très coûteux et peu pratiques.

Dans le domaine de la recherche de nouvelles sources laser dans l'UV, la possibilité d'obtenir des émissions laser à partir des transitions entre les configurations $4 f^{\mathrm{n}-1} 5 d$ et $4 f^{\mathrm{n}}$ des ions terres rares, introduits à l'état de dopant dans des matériaux diélectriques, suscite un grand intérêt. Ces transitions interconfigurationnelles ont pour caractéristiques d'être très efficaces, puisque permises à l'ordre dipolaire électrique, et de se présenter sous forme de bandes spectrales larges, ce qui pourrait permettre d'obtenir des émissions laser accordables.

Les ions terres rares étudiés actuellement sont principalement les ions $\mathrm{Ce}^{3+}, \mathrm{Pr}^{3+}$ et $\mathrm{Nd}^{3+}$. Les émissions laser potentielles de ces ions pourraient donner accès à des domaines spectraux complémentaires puisqu'elles pourraient couvrir, suivant le matériau hôte, plusieurs dizaines de nanomètres entre 280 et $450 \mathrm{~nm}$ pour l'ion $\mathrm{Ce}^{3+}$ ou entre 220 et $340 \mathrm{~nm}$ pour l'ion $\mathrm{Pr}^{3+}$. L'ion $\mathrm{Nd}^{3+}$ présente généralement trois bandes d'émission, d'environ $10 \mathrm{~nm}$ de large, autour de 190, 230, et $260 \mathrm{~nm}$.

À l'heure actuelle, seuls sont développés les cristaux dopés par l'ion $\mathrm{Ce}^{3+}$ qui peuvent donner des efficacités de l'ordre de $50 \%$ [1]. Les cristaux dopés $\operatorname{Pr}^{3+}$ n'ont pas encore donné lieu à un effet laser et sont en cours d'étude [2].

Élaboration et caractérisation des cristaux massifs et en couches minces pour l'optique

(C) EDP Sciences, 2003

http://dx.doi.org/10.1051/bib-sfo:2002806 
Ce document concerne plus particulièrement l'ion $\mathrm{Nd}^{3+}$ qui semble prometteur puisqu'une émission stimulée à $260 \mathrm{~nm}$ a déjà été mise en évidence pour $\mathrm{LiYF}_{4}: \mathrm{Nd}^{3+}$ après pompage direct de la bande $4 f^{2} 5 d$ à $157 \mathrm{~nm}$ [3]. Cet ion possède plusieurs niveaux d'énergie métastables dans la configuration fondamentale $4 f^{3}$ dont on pourrait tirer parti pour réaliser un pompage par absorptions successives de plusieurs photons. Ceci permettrait de bénéficier, pour le pompage, de sources usuelles telles que des diodes lasers ou des mini lasers pompés par diodes et d'obtenir ainsi un système compact. De plus, une telle excitation, mettant en jeu des photons moins énergétiques que dans le cas d'un pompage direct dans les états de la configuration excitée $4 f^{2} 5 d$, permettrait de réduire les problèmes de solarisation.

La spectroscopie approfondie de l'ion $\mathrm{Nd}^{3+}$ introduit à l'état de dopant dans différents matériaux diélectriques transparents dans l'UV (essentiellement des cristaux de fluorure) doit contribuer à obtenir une bonne connaissance des états de la configuration $4 f^{2} 5 d$ et à mesurer les sections efficaces de transition entre les états des configurations $4 f^{3}$ et $4 f^{2} 5 d$, de manière à déterminer les schémas de pompage les plus efficaces et les matériaux hôtes les mieux adaptés pour la réalisation d'une éventuelle source laser accordable dans l'UV.

Ce document présente les spectres d'absorption dans l'état excité et d'émission obtenus pour les matériaux suivants : les fluorures $\mathrm{LiYF}_{4}: \mathrm{Nd}^{3+}$ (1.6at.\%), $\mathrm{LiLuF}_{4}: \mathrm{Nd}^{3+} \quad$ (0.29at.\%), $\mathrm{BaY}_{2} \mathrm{~F}_{8}: \mathrm{Nd}^{3+}\left(1.05\right.$ at.\%), $\mathrm{Na}_{0.4} \mathrm{Y}_{0.6} \mathrm{~F}_{2.2}: \mathrm{Nd}^{3+}$ (5at.\%) et le chlorure $\mathrm{Cs}_{2} \mathrm{NaYCl}_{6}: \mathrm{Nd}^{3+}$ (5at.\%).

\section{RÉSULTATS EXPÉRIMENTAUX ET INTERPRÉTATION}

\subsection{Spectres d'absorption dans l'état excité à partir du niveau ${ }^{4} \mathbf{F}_{3 / 2}$}

Pour qu'une excitation faisant intervenir deux photons soit efficace, la première étape (absorption du premier photon) doit aboutir à un niveau de durée de vie importante. Or les niveaux métastables de la configuration $4 f^{3}$ ont été largement étudiés, leurs positions en énergie, leurs durées de vie et l'énergie optimale du photon à mettre en jeu pour les peupler de manière efficace sont parfaitement connues. Les niveaux métastables ${ }^{4} \mathrm{D}_{3 / 2}$ et ${ }^{4} \mathrm{~F}_{3 / 2}$ sont intéressants, le premier parce qu'il se situe à mi-hauteur en énergie entre le niveau fondamental ${ }^{4} \mathrm{I}_{9 / 2}$ et le bas de la bande $4 f^{2} 5 d$, le pompage en deux étapes pourrait alors ne nécessiter qu'une seule source, le second parce qu'il est facilement accessible par pompage par diodes laser (Fig. 1).

La deuxième étape nécessite une étude puisque l'énergie optimale pour le second photon n'est pas connue. Pour cela, des spectres d'absorption dans l'état excité sont réalisés. Cette expérience est une expérience de type pompe-sonde. Le faisceau pompe porte les ions $\mathrm{Nd}^{3+}$ dans l'état métastable, le faisceau sonde permet d'évaluer l'efficacité d'absorption du second photon en fonction de son énergie.

La figure 2 schématise le dispositif expérimental utilisé pour mesurer l'absorption à partir du niveau ${ }^{4} \mathrm{~F}_{3 / 2}$. Un laser à colorant émettant à environ $590 \mathrm{~nm}$ permet de peupler le niveau ${ }^{4} \mathrm{G}_{5 / 2}$ ou ${ }^{4} \mathrm{G}_{7 / 2}$ puis par relaxation multiphonon l'état ${ }^{4} \mathrm{~F}_{3 / 2}$. Le faisceau sonde est issu d'une lampe deutérium (185$300 \mathrm{~nm}$ ). L'intensité du faisceau sonde est mesurée en présence ou non du faisceau pompe par un monochromateur Jobin-Yvon H20 suivi d'un photomultiplicateur Hamamatsu R1477.

Les spectres obtenus sont représentés sur la figure $\underline{3}$. La bande autour de $190 \mathrm{~nm}$ n'a pas pu être enregistrée entièrement du fait de l'absorption des photons par l'air lorsque la longueur d'onde est inférieure à $190 \mathrm{~nm}$.

Les sections efficaces d'absorption à partir du niveau ${ }^{4} \mathrm{~F}_{3 / 2}$ sont de l'ordre de $10^{-18} \mathrm{~cm}^{2}$, valeur caractéristique des transitions entre configurations $4 f^{3}$ et $4 f^{2} 5 d$ permises à l'ordre dipolaire électrique. L'absorption est particulièrement efficace pour le cristal $\mathrm{Cs}_{2} \mathrm{NaYCl}_{6}: \mathrm{Nd}^{3+}$ entre 215 et $270 \mathrm{~nm}$. Pour $\mathrm{LiYF}_{4}: \mathrm{Nd}^{3+}, \mathrm{LiLuF}_{4}: \mathrm{Nd}^{3+}, \mathrm{BaY}_{2} \mathrm{~F}_{8}: \mathrm{Nd}^{3+}$, la longueur d'onde la plus avantageuse pour le second photon se situe entre 210 et $225 \mathrm{~nm}$. Le spectre de $\mathrm{Na}_{0.4} \mathrm{Y}_{0.6} \mathrm{~F}_{2.2}: \mathrm{Nd}^{3+}$ est moins structuré compte tenu de la nature désordonnée de ce cristal : il ne présente pas réellement de maximum, les sections efficaces d'absorption dans l'état excité sont plus faibles mais restent du même ordre de grandeur que pour les autres fluorures. 


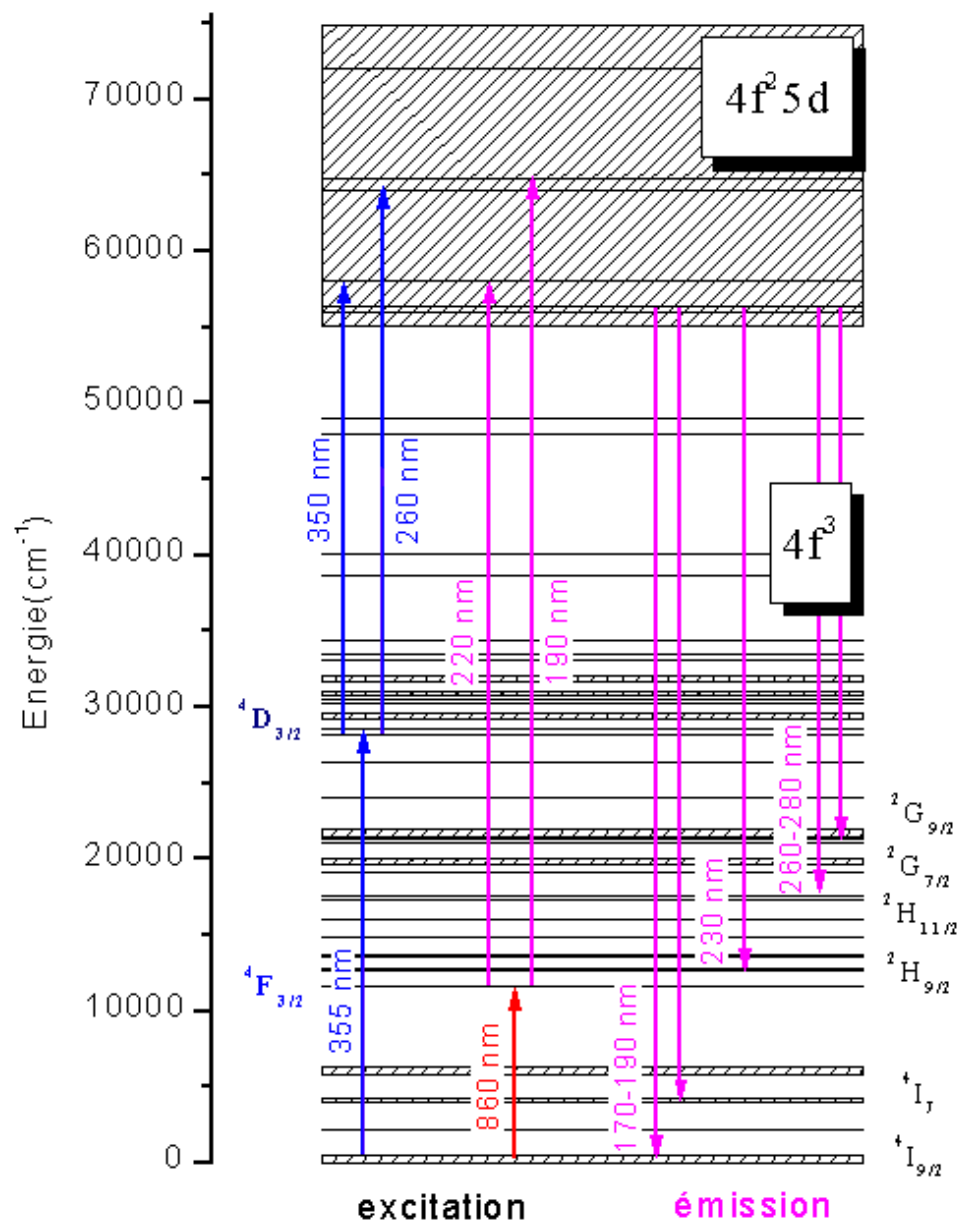

FIG. 1. Niveau d'énergie de l'ion $\mathrm{Nd}^{3+}$ dans Nd:LiYF 4 et transitions étudiées.

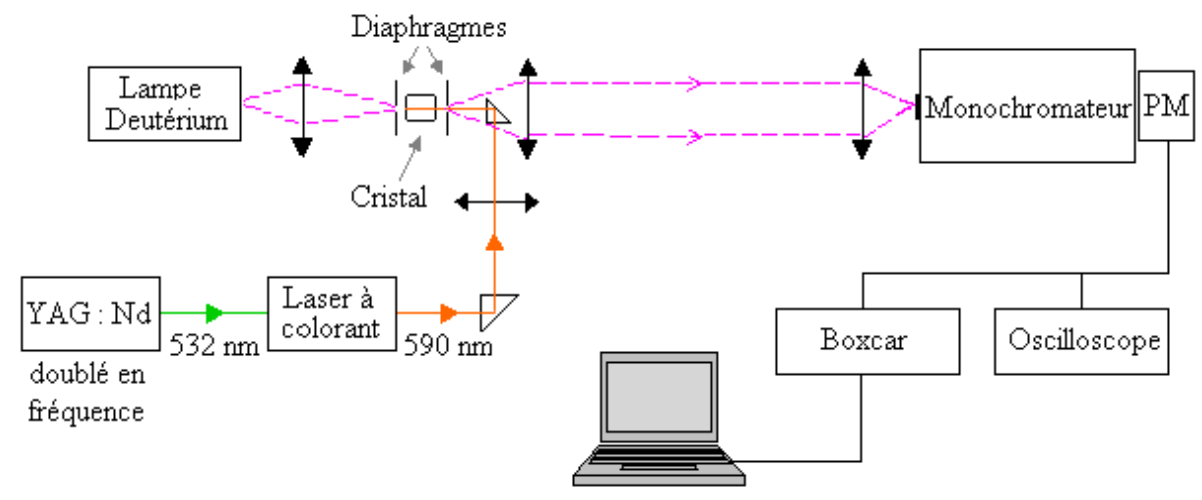

FIG. 2. Dispositif expérimental pour l'enregistrement des spectres d'absorption dans l'état excité à partir du niveau ${ }^{4} \mathrm{~F}_{3 / 2}$. 


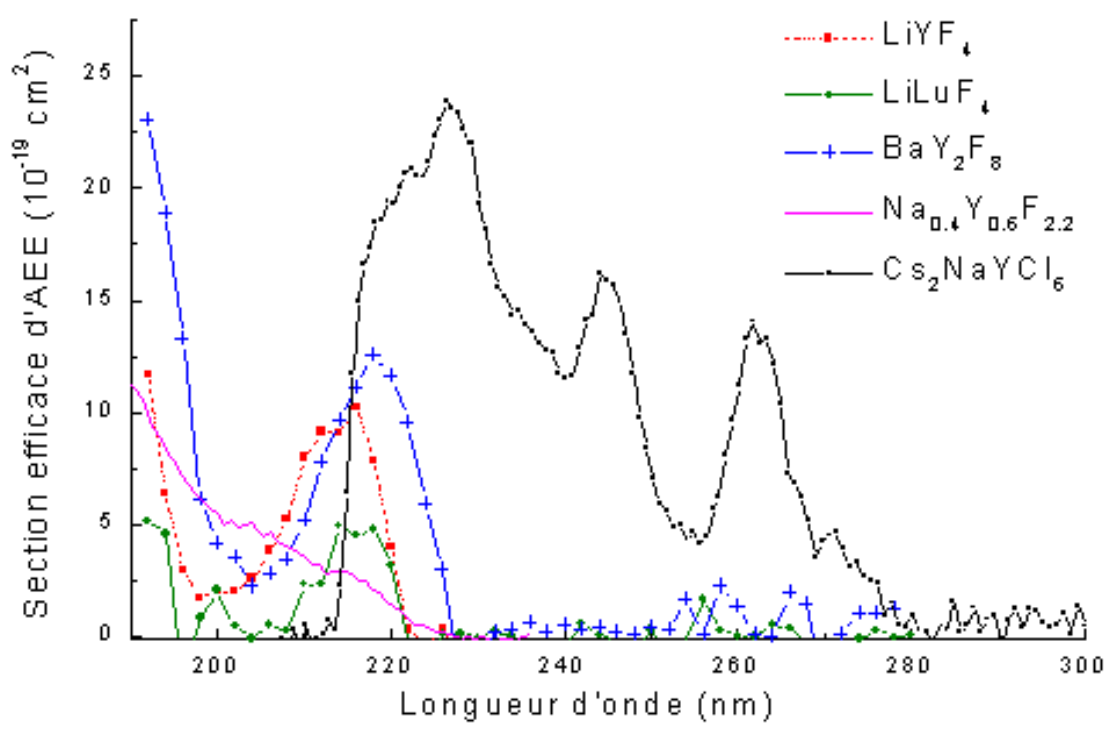

FIG. 3. Spectres d'absorption dans l'état excité à partir du niveau ${ }^{4} F_{3 / 2}$.

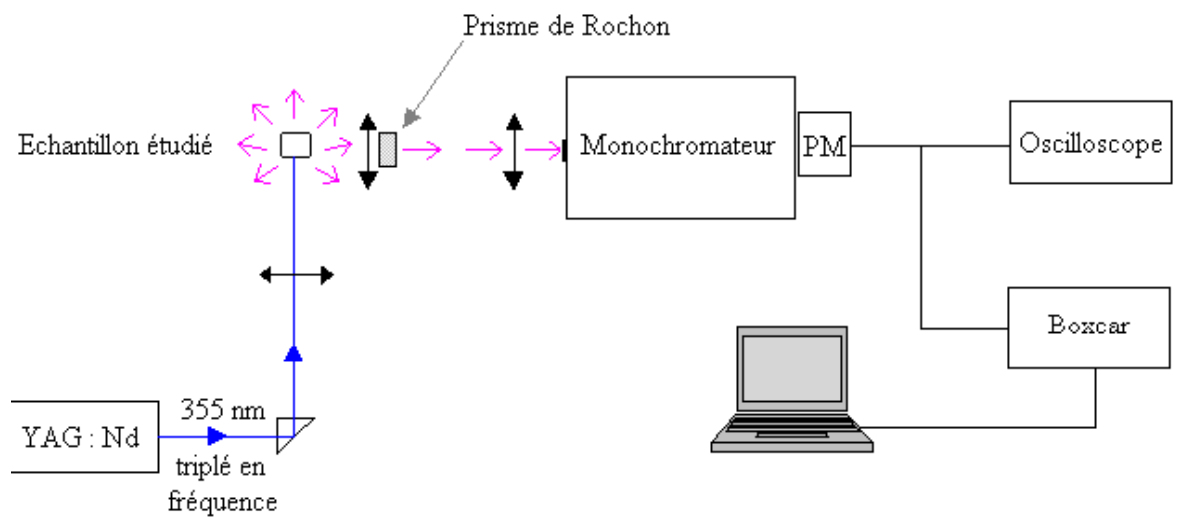

FIG. 4. Dispositif expérimental pour l'enregistrement des spectres d'absorption.

Des tests d'absorption dans l'état excité à partir du niveau ${ }^{4} \mathrm{D}_{3 / 2}$ ont montré des sections efficaces de l'ordre de $10^{-20} \mathrm{~cm}^{2}$ [4] , soit de deux ordres de grandeur inférieurs aux valeurs attendues.

\subsection{Spectres d'émission obtenus après excitation à $355 \mathrm{~nm}$}

Bien qu'une excitation à deux photons de longueur d'onde $355 \mathrm{~nm}$, utilisant le niveau métastable ${ }^{4} \mathrm{D}_{3 / 2}$ comme niveau intermédiaire, soit assez inefficace pour peupler le bas de la bande $4 f^{2} 5 d$, elle est néanmoins suffisante pour observer les bandes larges caractéristiques des émissions $4 f^{2} 5 d \rightarrow 4 f^{3}$ (Fig. 1).

Le dispositif expérimental utilisé est schématisé sur la figure $\underline{4}$. La source d'excitation est un laser YAG: $\mathrm{Nd}^{3+}$ triplé en fréquence. Le système de détection est constitué d'un monochromateur Hilger \& Watt, Monospek 1000, suivi d'un photomultiplicateur EMI 9789Q. Un prisme de Rochon disposé derrière l'échantillon permet de recueillir les émissions polarisées des cristaux anisotropes (uniaxes : $\mathrm{LiYF}_{4}, \mathrm{LiLuF}_{4}$ ou biaxe : $\mathrm{BaY}_{2} \mathrm{~F}_{8}$ ).

La figure $\underline{5}$ présente les spectres enregistrés entre 190 et $300 \mathrm{~nm}$. Seule l'émission du Nd ${ }^{3+}$ dans le chlorure $\mathrm{Cs}_{2} \mathrm{NaYCl}_{6}$ n'a pas été observée. Dans ce cristal, la position en énergie du bas de la bande $4 f^{2} 5 d$ est trop proche des niveaux supérieurs de la configuration $4 f^{3}$, ce qui a pour conséquence de privilégier les désexcitations non radiatives par rapport aux émissions radiatives. 

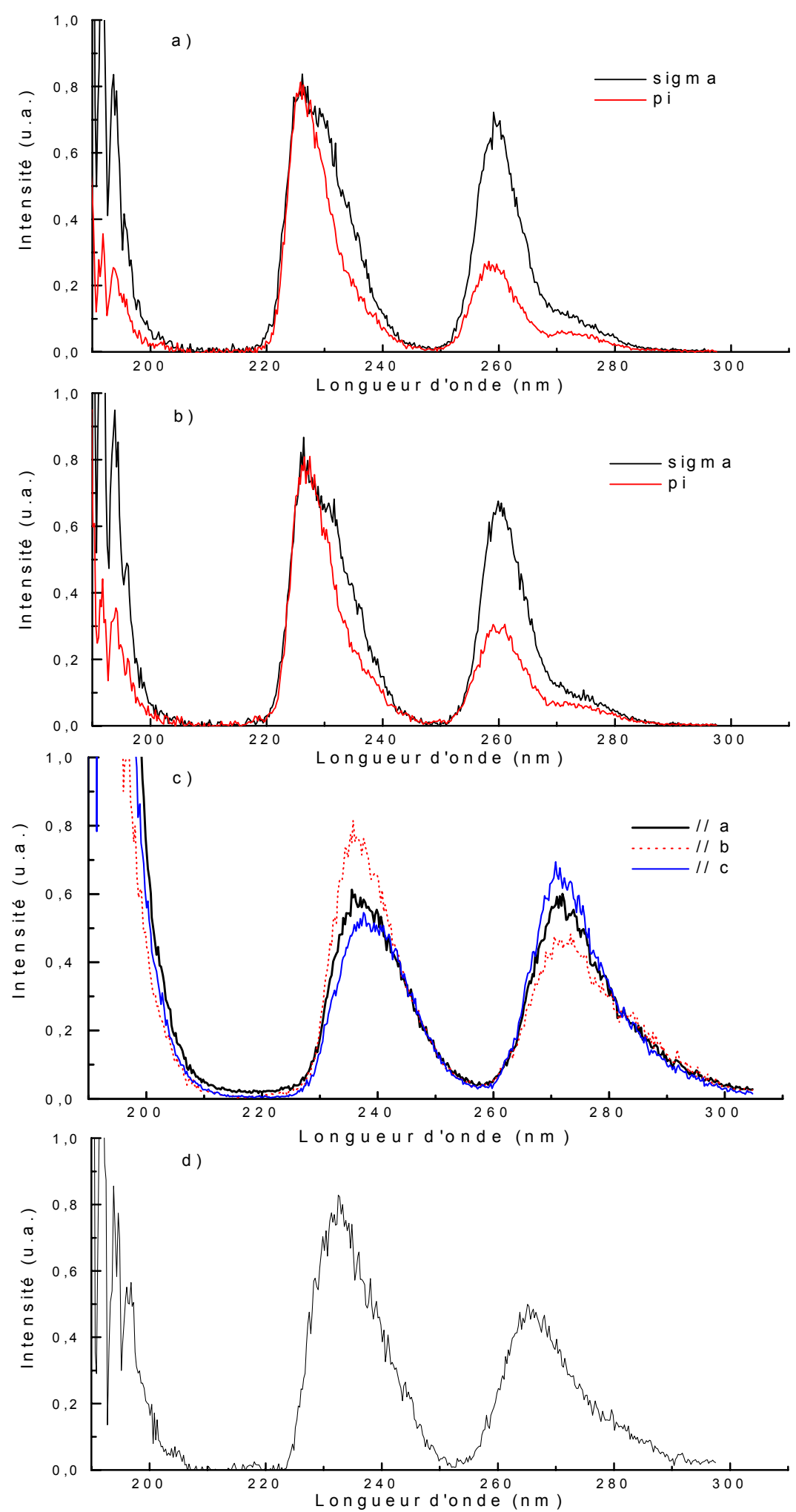

FIG. 5. Spectres d'émission de (a) $\mathrm{LiYF}_{4}: \mathrm{Nd}^{3+}$, (b) $\mathrm{LiLuF}_{4}: \mathrm{Nd}^{3+}$, (c) $\mathrm{BaY}_{2} \mathrm{~F}_{8}: \mathrm{Nd}^{3+}$ et (d) $\mathrm{Na}_{0.4} \mathrm{Y}_{0.6} \mathrm{~F}_{2.2}: \mathrm{Nd}^{3+}$.

Les spectres sont très ressemblants pour les différents fluorures étudiés, surtout pour les cristaux $\mathrm{LiYF}_{4}: \mathrm{Nd}^{3+}$ et $\mathrm{LiLuF}_{4}: \mathrm{Nd}^{3+}$ qui ont la même structure. Ils montrent trois bandes polarisées. La bande à $190 \mathrm{~nm}$ n'a pu être enregistrée entièrement à cause de l'absorption de l'air dans ce domaine spectral, on constate néanmoins que son intensité est plus importante que celles des deux autres bandes. 


\section{CONCLUSION}

Les spectres d'absorption dans l'état excité ainsi que les spectres d'émission en lumière polarisée présentés ici constituent des données expérimentales intéressantes pour des travaux de modélisation des transitions $4 f^{3} \leftrightarrow 4 f^{2} 5 d$ de l'ion $\mathrm{Nd}^{3+}$.

L'absorption dans l'état excité à partir du niveau ${ }^{4} \mathrm{~F}_{3 / 2}$ est très efficace, contrairement à celle mesurée à partir du niveau ${ }^{4} \mathrm{D}_{3 / 2}$, ce qui est prometteur pour la conception de lasers accordables dans l'ultraviolet pompés par absorption successive de deux photons.

\section{REMERCIEMENTS}

Nous remercions nos collaborateurs du contrat INTAS n 97-787 qui nous ont fourni les fluorures étudiés, ainsi que P. Tanner, City University of Hong Kong, pour le chlorure $\mathrm{Cs}_{2} \mathrm{NaYCl}_{6}: \mathrm{Nd}^{3+}$.

\section{RÉFÉRENCES}

[1] A.J.S. McGonigle, S. Girard, D.W. Coutts et R. Moncorgé, Elect. Lett. 35 (1999) 1640.

[2] S. Nicolas, Y. Guyot, M.-F. Joubert et E. Descroix, in Actes du $5^{\text {ème }}$ colloque sur les sources cohérentes et incohérentes $U V, V U V$ et $X$-applications et développements récents, J. Phys IV 11 (2001) 57 ; S. Nicolas, M. Laroche, S. Girard, R. Moncorgé, Y. Guyot, M.-F. Joubert, E. Descroix et A.G. Petrosyan, J. Phys. Cond. Matter 11 (1999) 7937.

[3] A.C. Cefalas, M.A. Dubinskii, E. Sarantopoulou, R.Yu. Abdulsabirov, S.L. Korableva, A.K. Naumov, V.V. Shemashko et C.A. Nicolaides, Laser Chem. 13 (1993) 143.

[4] Y. Guyot, S. Guy, J-Y. Rivoire, A.M. Tkachuk, I.K. Razumova, C. Pédrini et M.-F. Joubert, Rencontre ILLUM'99, supplément à la revue Le vide: science, technique et applications (sept. 1999) 13 ; Y. Guyot, S. Guy et M.-F. Joubert, J. Alloys and Compounds 323-324 (2001) 722. 